OPEN ACCESS

Edited by:

Elena Vegni,

University of Milan, Italy

Reviewed by:

Rita Berger

University of Barcelona, Spain

Serena Barello,

Catholic University of the Sacred

Heart, Italy

*Correspondence:

Simone Garzon

simone.garzon@univr.it

orcid.org/0000-0002-5840-699X

tThese authors have contributed equally to this work

Specialty section:

This article was submitted to

Psychology for Clinical Settings,

a section of the journal

Frontiers in Psychology

Received: 24 November 2020 Accepted: 03 March 2021

Published: 09 April 2021

Citation:

Del Piccolo L, Donisi V, Raffaelli R, Garzon S, Perlini C, Rimondini M,

Uccella S, Cromi A, Ghezzi F,

Ginami M, Sartori E, Ciccarone $F$, Scambia G and Franchi M (2021) The Psychological Impact of COVID-19 on Healthcare Providers in Obstetrics: A

Cross-Sectional Survey Study.

Front. Psychol. 12:632999. doi: 10.3389/fpsyg.2021.632999

\section{The Psychological Impact of} COVID-19 on Healthcare Providers in Obstetrics: A Cross-Sectional Survey Study

Lidia Del Piccolo ${ }^{1 \dagger}$, Valeria Donisi ${ }^{1 \dagger}$, Ricciarda Raffaelli ${ }^{2}$, Simone Garzon ${ }^{2 *}$, Cinzia Perlini ${ }^{1}$, Michela Rimondini ${ }^{1}$, Stefano Uccella ${ }^{2}$, Antonella Cromi ${ }^{3}$, Fabio Ghezzi ${ }^{3}$, Maddalena Ginami ${ }^{4}$, Enrico Sartori ${ }^{4}$, Francesca Ciccarone ${ }^{5}$, Giovanni Scambia ${ }^{5}$ and Massimo Franchi ${ }^{2}$

\footnotetext{
${ }^{1}$ Department of Neuroscience, Biomedicine and Movement Sciences, University of Verona, Verona, Italy, ${ }^{2}$ Department of Obstetrics and Gynecology, AOUI Verona, University of Verona, Verona, Italy, ${ }^{3}$ Department of Obstetrics and Gynecology, "Filippo Del Ponte" Hospital, University of Insubria, Varese, Italy, ${ }^{4}$ Department of Obstetrics and Gynecology, University of Brescia, Brescia, Italy, ${ }^{5}$ Division of Gynecologic Oncology, Department of Obstetrics and Gynecology, Catholic University of the Sacred Heart, Rome, Italy
}

Objective: To assess the psychological distress of healthcare providers (HCPs) working in the field of obstetrics during the coronavirus disease 2019 (COVID-19) pandemic and to identify factors associated with psychological distress at the individual, interpersonal, and organizational level.

Design: Cross-sectional survey study.

Setting: Four University hospitals in Italy.

Participants: HCPs working in obstetrics, including gynecologists, residents in gynecology and obstetrics, and midwives.

Methods: The 104-item survey Impatto PSIcologico COVID-19 in Ostetricia (IPSICO) was created by a multidisciplinary expert panel and administered to HCPs in obstetrics in May 2020 via a web-based platform.

Main Outcome Measures: Psychological distress assessed by the General Health Questionnaire-12 (GHQ-12) included in the IPSICO survey.

Results: The response rate to the IPSICO survey was 88.2\% (503/570), and that for GHQ-12 was 84.4\% (481/570). Just over half (51.1\%; 246/481) of the GHQ-12 respondents reported a clinically significant level of psychological distress (GHQ-12 $\geq 3)$. Psychological distress was associated with either individual (i.e., female gender, stressful experience related to COVID-19, exhaustion, and the use of dysfunctional coping strategies), interpersonal (i.e., lower family support, limitations in interactions with colleagues), and organizational (i.e., reduced perception of protection by personal protective equipment, perceived delays on updates and gaps in information on the pandemic) factors in dealing with the pandemic. 
Conclusions: Results confirm the need for monitoring and assessing the psychological distress for HCPs in obstetrics. Interventions at the individual, interpersonal, and organizational level may relieve the psychological distress during the COVID-19 pandemic and foster resilience skills in facing emotional distress.

Keywords: health care providers, COVID-19, obstetrics, psychological distress, GHQ-12, coping strategies, stress

\section{INTRODUCTION}

Since the worldwide outbreak of severe acute respiratory syndrome coronavirus 2 (SARS-CoV-2) in March 2020 (WHO, 2020), healthcare systems and healthcare providers (HCPs) have been placed under extreme pressure and challenges. Different authors outlined the psychological impact of this condition, recommending tailored psychosocial interventions to preserve the well-being of HCPs and the quality of healthcare provided to the patients (Galli et al., 2020; Greenberg et al., 2020; Lai et al., 2020; Nie et al., 2020; Preti et al., 2020; Shaukat et al., 2020; Shreffler et al., 2020; Yao et al., 2020).

North Italian regions were the first in Europe to face the coronavirus disease 2019 (COVID-19) pandemic and the associated pressure on the healthcare system and HCPs (Alfieri et al., 2020; Armocida et al., 2020; Oliva et al., 2020). High levels of burnout, psychological distress, and psychosomatic symptoms were observed in physicians, nurses, and other professionals at the peak of the pandemic (Barello et al., 2020a,b; Giusti et al., 2020; Marton et al., 2020). Although HCPs working with COVID-19 patients reported a higher level of stress, depressive and anxiety symptoms, burnout, and post-traumatic stress disorders than other HCPs (Babore et al., 2020; Di Tella et al., 2020; Trumello et al., 2020), the emergency might have amplified preexisting vulnerability factors for psychological distress, regardless of direct or indirect management of COVID19 patients. Therefore, baseline risk may help identify those HCPs who are more susceptible to adverse psychological impact of the COVID-19 pandemic. In this regard, HCPs who work in obstetrics are among those with a noticeable baseline risk for burnout and distress (Becker et al., 2006; Govardhan et al., 2012; Wahlberg et al., 2017; Bourne et al., 2019; Slade et al., 2020).

To the best of our knowledge, the data on COVID-19related psychosocial distress in HCPs in obstetrics are limited, with the exception of a UK-wide study, which identified a high prevalence of depression and anxiety among obstetricians and gynecologists (Shah et al., 2020). HCPs working in obstetrics and gynecology experienced common and unique challenges during the COVID-19 pandemic. Similar to other HCPs, HCPs in obstetrics also had to adjust to the implementation of infection control measures, dedicated "emergency protocols," personal risk of exposure to infection, as well as concerns about the provision and use of personal protective equipment (PPE) (Alfieri et al., 2020; Armocida et al., 2020; Oliva et al., 2020). Moreover, HCPs in obstetrics faced specific challenges: the limited rescheduling of obstetrics care, the uncertainties about the potential of vertical transmission of SARS-CoV-2, the management of SARS-CoV2-positive women during labor, the care of psychologically vulnerable patients without the involvement of the partner, and an increased rate of intrauterine fetal death due to reduced use of emergency service (Boelig et al., 2020; Dell'Utri et al., 2020; Franchi et al., 2020; Green et al., 2020; Qiao, 2020; Vafaei et al., 2020; Yalçin Bahat et al., 2020).

Based on this background, we investigated the psychological distress of HCPs working in obstetrics during the current pandemic in different Italian hospitals. This study aimed to identify HCPs with psychological distress and explore potentially associated factors at the individual, interpersonal, and organizational levels. The "socioecological" model proposed by Winkel et al. (2019) explaining how resilience grows in obstetrician-gynecologists was adopted to build up the Impatto PSIcologico COVID-19 in Ostetricia (IPSICO) survey. This model is based on grounded theory and showed that resilience emerges as "a capacity to connect authentically with the work that is influenced by personal and professional surroundings" and underlines the importance of "both individual and collective actions in promoting an environment in which physicians thrive." Therefore, in our study, we decided to analyze how individual response to adversity (i.e., level of perceived distress) was related either to personal factors (age, gender, psychological well-being before COVID-19 pandemic, perceived risk of infection, coping strategies, professional role, the experience of quarantine or self-isolation, and stressful events related and not related to COVID 19), quality of connections to others inside and outside professional activity (type and quality of support received by family, friends or others, and by colleagues), or to contextual and organizational factors (measures contributing to a greater sense of security, aspects related to the greatest stress, availability of organizational, and clinical protocols to deal with the pandemic). By using this approach, we aim to establish which are the most relevant intervening aspects contributing to the emotional burden of HCPs during the current pandemic and to define the type of intervention that is more appropriate at each level (individual, interpersonal, and organizational). A better understanding of the level at which influencing factors affect the professional well-being of HCPs in obstetrics is highly relevant to guide more appropriate interventions to manage distress and its negative consequences.

\section{MATERIALS AND METHODS Study Population and Study Design}

Target respondents were all HCPs (gynecologists, residents in gynecology and obstetrics, and midwives) working at four Italian University hospitals (the University of Verona, the Catholic 
University of the Sacred Heart of Rome, the University of Insubria, and the University of Brescia) accruing to a total of 570 HCPs in obstetrics. HCPs were invited by e-mail to complete the IPSICO survey between May 15, 2020, and May 31, 2020. The electronic invitation included the study presentation and the link to the survey located at a web-based platform. Each center provided the complete list of institutional e-mail addresses of target respondents. The survey was administered in the Italian language. Participation was voluntary and anonymous, and no remuneration was offered to respondents. HCPs were reminded up to 3 times by e-mail whether they were willing or not to take part in the survey.

The study was approved by the human research ethics committee of the University of Verona (2020-UNVRCLE0143469). All participants gave informed consent for study participation and anonymized data collection and analysis for research purposes prior to accessing and completing the survey. There was no funding for the design and conduct of the study.

\section{The IPSICO Survey}

The IPSICO survey was designed and validated by a panel of trainees, specialty tutors, medical educationalists in obstetrics and gynecology, and clinical psychologists of the University of Verona. The survey resulted in a 104-item battery investigating the sociodemographic and professional characteristics of HCPs in obstetrics, the risk appraisal along with perceived social support and coping strategies, the perceived organizational support and changes in the work organization and climate, the emotional impact of COVID-19, and the impact of COVID-19 on the professional life, along with a measure of psychological distress. The survey was composed of validated psychological questionnaires and items tailored to obstetrics practice and COVID-19. Psychological questionnaires were already validated in the Italian language, such as the short version of the Coping Orientation to Problems Experienced (Brief-COPE) (Carver et al., 1989; Carver, 1997; Coolidge et al., 2000) questionnaire, and the General Health Questionnaire-12 (GHQ-12) (Piccinelli et al., 1993; Politi et al., 1994; Goldberg et al., 1997). Newly developed items were limited to exploring sociodemographic, obstetrics, and COVID-19-related factors.

\section{Variables}

The primary outcome was the presence or absence of clinically significant psychological distress in HCPs. The psychological distress level of HCPs was assessed by the validated Italian version of the GHQ-12 (Piccinelli et al., 1993; Politi et al., 1994; Goldberg et al., 1997), a widely used screening instrument for psychological distress. The GHQ-12 was analyzed based on the method proposed by Piccinelli et al. (1993) (all the 12 items at a 4-level scale of the GHQ-12 survey were scored as $0,0,1,1)$. A Cronbach $\alpha$ of 0.84 indicated a satisfactory internal consistency of the GHQ-12 in our sample (Politi et al., 1994). HCPs reporting a GHQ-12 score $\geq 3$ were considered positive for the presence of clinically significant psychological distress.

A series of individual, interpersonal, and organizational factors have been used to describe the sample and evaluate their associations with the GHQ-12.
Sociodemographic variables included age (continuous variable), gender (i.e., male, female), marital status (i.e., married/cohabitant, separated/widowed, unmarried), family composition (i.e., single, couple, couple with children, two or more adults not familiar), and presence of old parents (i.e., yes, no). Professional variables investigated the professional role (i.e., specialized doctor, trainee doctor, midwife) and the years of work (continuous variable).

Coping strategies were evaluated using the Brief-COPE (Carver et al., 1989; Carver, 1997). The Brief-COPE is composed of 28 items describing different coping strategies self-evaluated by respondents on a 4-point Likert scale ranging from 1 ("not doing it at all") to 4 ("doing it a lot"). The coping strategies were grouped into emotions-focused (Cronbach $\alpha=0.69$ ), problemsfocused (Cronbach $\alpha=0.66$ ), and dysfunctional (Cronbach $\alpha=$ 0.78 ) coping strategies (Coolidge et al., 2000).

Using categorical variables (i.e., yes, no), the survey has evaluated if participants underwent a quarantine period, experienced a period of self-isolation, or experienced stressful events related and not related to COVID-19. Moreover, categorical variables were used to investigate the adoption of a shift strategy and the availability of organizational and clinical protocols to deal with the emergency problem.

All other variables regarding individual (i.e., psychological well-being before COVID-19; perceived risk of infection and death; level of professional satisfaction before the pandemic; other negative perceptions and feelings related to work"exhaustion," "weight of professional role," "consideration to abandon the professional role," "working as duty"), interpersonal (i.e., support received by family, friends, trustworthy people, and colleagues; changes in the rules of interaction with colleagues and in the quality of relationship with patients), and organizational factors (i.e., protection by PPE; efficacy of patient triage on admission; utility of the shift strategy; receiving timely and complete information on the pandemic; reduction in the quality of obstetric service and change in perceived obstetric risk; level of involvement as an active part in the reorganization) have been self-evaluated by HCPs on a 10-point Likert scale ranging from 1 ("not at all") to 10 ("extremely").

Finally, the respondents were asked to select the aspects related to the greatest stress during COVID-19, the factors associated with a sense of security, the prevailing sensations in the relationship with the patient, and the prevalent feelings toward colleagues. Respondents could give more than one answer selecting the most corresponding ones to their personal experiences.

Further details of the IPSICO survey and details on all the survey variables were reported and described elsewhere (Del Piccolo et al., 2020).

\section{Statistical Analysis}

Descriptive statistics were used to summarize variables collected in the IPSICO questionnaire and the main characteristics of the study population. For the IPSICO questionnaire analysis, no exclusion criteria were used. Descriptive statistics were expressed with mean and standard deviation (SD) for variables with a normal distribution. Non-normally distributed and ordinal variables were described with median and interquartile range 
(IQR); nominal variables were summarized with numbers and percentages. The GHQ-12 and Brief-COPE's reliability in our sample was estimated on the observed correlations of the items with each other and expressed using the Cronbach $\alpha$. The MannWhitney $U$-test was used to compare two independent groups, and the Kruskal-Wallis test was adopted in the case of three or more categories. Categorical data were analyzed with the $\chi^{2}$ test or Fisher exact test as appropriate. All reported $p$-values were two-sided, and significance was considered at $p<0.05$. In the case of post-hoc pairwise comparisons with three or more groups, $p$-values were adjusted using the Bonferroni correction.

After identifying HCPs with psychological distress (i.e., GHQ12 score $\geq 3$ ), associated factors at the individual, interpersonal, and organizational levels have been explored using (i) logistic regression models and (ii) comparing HCPs with GHQ-12 score $\geq 3$ with those with GHQ-12 score $<3$ in terms of perceived aspects related to the greatest stress and of interpersonal and organizational factors.

Logistic regression models were used to investigate the univariate association between clinically significant distress (GHQ-12 score $\geq 3$ ) and different variables. Variables associated with the dependent variable in univariate analysis were included in a multivariable model, which was developed starting with a backward stepwise selection to eliminate less relevant variables and then using a hierarchical method for the final choice of predictors. The corrected Akaike information criterion was used to select the model (Ratner, 2010; Hosmer et al., 2013; Chowdhury and Turin, 2020). After defining the multivariable logistic regression model with fixed coefficients, multilevel logistic regression analysis was used to explore the multilevel structure of data related to the aggregation of HPCs within University hospitals (Raudenbush and Bryk, 2002). Comparisons between the model with fixed coefficients and multilevel models were made using the corrected Akaike information criterion (Tabachnick et al., 2019). This further analysis was performed because of the fact that our survey data had an inherent multilevel structure: HCPs within University hospitals (van Oyen, 2009). Therefore, the defined multivariable logistic regression model with fixed coefficients would not have completely corrected for between-group (University hospitals) differences, potentially relevant due, for example, to the different incidence of COVID-19 cases in the four geographic areas. The multilevel approach allows exploring effects that vary by hospitals, studying characteristics contributing to this differential effect, and identifying individual-group interaction effects (Cohen et al., 2013). All reported $p$-values were two-sided, and significance was considered at $p<0.05$.

Data analysis was performed using IBM SPSS Statistics 23.0, Armonk, NY.

\section{RESULTS}

\section{Sociodemographic, Professional Characteristics, and COVID-19 Impact in the Study Sample}

Of 570 invited HCPs, 503 (88.2\%) answered the IPSICO survey. Sociodemographic characteristics of the entire study population are summarized in Table 1, overall and stratified by center. The median age of respondents was 34 years (IQR $=29-46$ years), and $83.7 \%$ of HCPs were female. Midwives represented more than one-third of respondents (38\%), followed by trainees $(33.6 \%)$ and specialized medical doctors (28.4\%). Overall, the entire study sample reported a median work experience in the current role of 5 years (IQR $=2-18$ years). Regarding family composition, most respondents answered to have a partner, and $38.8 \%$ reported to live with children and $7.7 \%$ with old parents; $77.7 \%$ of HCPs reported to live with someone else. Psychological well-being before the COVID-19 pandemic was self-evaluated as high, with a median value of 8 (IQR $=7-8)$ on a Likert scale of $1=$ "very bad" to $10=$ "very good."

Concerning the impact of COVID-19, although only $10.1 \%$ of HCPs experienced a quarantine period, almost one-third of them (32.2\%) decided to undergo a period of self-isolation, and 51.9\% of respondents experienced a stressful event related to COVID19. The perceived risk of infection was reported higher than 5 , on a scale of $1-10$, by half of the respondents (IQR $=5-8$ ); conversely, the perceived risk of death in the case of infection was lower, with a median of $3(\mathrm{IQR}=2-4)$.

\section{Prevalence of Psychological Distress}

Four hundred eighty-one of $570 \mathrm{HCPs}$ completed all items of the GHQ-12, resulting in a response rate of $84.4 \%$. The impact of COVID-19 on psychological distress assessed with the GHQ-12 is summarized in Figure 1. GHQ-12 $\geq 3$ was observed in 51.1\% of respondents (246/481HCPs).

\section{Factors Associated With Psychological Distress at the Individual, Interpersonal, and Organizational Levels \\ Factors Associated With Psychological Distress in Univariate and Multivariable Logistic Regression Analyses}

Table 2 shows the results of the univariate logistic regression analysis investigating the association between psychological distress (GHQ-12 $\geq 3$ ) and individual, interpersonal, and organizational factors derived from the IPSICO questionnaire. Variables univariately associated with a GHQ-12 score equal to or higher than 3 were included in the multivariable logistic regression analysis together with age. Individual factors independently associated with a GHQ-12 score $\geq 3$ were gender, the experience of stressful events related to the ongoing pandemic, dysfunctional coping score (Table 3 reports the details regarding the Brief-COPE items composing the dysfunctional coping score) (Carver et al., 1989; Carver, 1997; Coolidge et al., 2000), and perceived exhaustion from work. Interpersonal aspects were the perceived support received from the family and the limited interaction with colleagues. Organizational factors were the perceived protection by PPE and the possibility to receive timely and complete information on the pandemic (Table 4). Multilevel logistic regression models, fitted allowing parameters to vary between the University hospitals (random effect), were compared with the multivariable logistic regression model with fixed coefficients reported in Table 4. The comparison, made with the corrected Akaike 
TABLE 1 | Sociodemographic and professional characteristics of the study population $(n=503)$.

\begin{tabular}{|c|c|c|c|c|c|c|}
\hline Variable & $\begin{array}{c}\text { Overall } \\
503(100 \%)\end{array}$ & $\begin{array}{c}\text { Brescia } \\
185(36.8 \%)\end{array}$ & $\begin{array}{c}\text { Rome } \\
111(22.1 \%)\end{array}$ & $\begin{array}{c}\text { Varese } \\
82(16.3 \%)\end{array}$ & $\begin{array}{c}\text { Verona } \\
125(24.9 \%)\end{array}$ & $p$-value \\
\hline Age, median (IQR) & $34(29-46)$ & $39(30-51)^{a}$ & $32(28-38)^{b}$ & $35(29.75-47.25)^{\mathrm{a}, \mathrm{b}}$ & $31(28-41.5)^{b}$ & $<0.001$ \\
\hline Gender, $n(\%)$ & & & & & & $<0.001$ \\
\hline Female & $421(83.7)$ & $161(87.0)^{\mathrm{a}}$ & $77(69.4 \%)^{b}$ & $69(84.1)^{a, b}$ & $114(91.2)^{\mathrm{a}}$ & \\
\hline Marital status, $n(\%)$ & & & & & & 0.06 \\
\hline Unmarried & $205(40.8)$ & $68(36.8)$ & $53(47.7)$ & $26(31.7)$ & $58(46.4)$ & \\
\hline Family composition, $n(\%)$ & & & & & & $<0.001$ \\
\hline Single & $112(22.3)$ & $33(17.8)^{a}$ & $32(28.8)^{a}$ & $17(20.7)^{\mathrm{a}}$ & $30(24.0)^{\mathrm{a}}$ & \\
\hline Couple & $124(24.7)$ & $36(19.5)^{a}$ & $39(35.1)^{b}$ & $21(25.6)^{a, b}$ & $28(22.4)^{a, b}$ & \\
\hline Couple with children & $195(38.8)$ & $87(47.0)^{\mathrm{a}}$ & $24(21.6)^{b}$ & $40(48.8)^{\mathrm{a}}$ & $44(35.2)^{a, b}$ & \\
\hline Specialized doctor & $143(28.4)$ & $42(22.7)^{\mathrm{a}}$ & $54(48.6)^{b}$ & $35(42.7)^{\mathrm{b}}$ & $12(9.6)^{c}$ & \\
\hline Trainee doctor & $169(33.6)$ & $35(18.9)^{\mathrm{a}}$ & $55(49.5)^{b}$ & $24(29.3)^{a, c}$ & $55(44.0)^{\mathrm{b}, \mathrm{c}}$ & \\
\hline Midwife & $191(38.0)$ & $108(58.4)^{\mathrm{a}}$ & $2(1.8)^{\mathrm{b}}$ & $23(28.0)^{c}$ & $58(46.4)^{a}$ & \\
\hline Years of work experience in the current role, median (IQR) & $5(2-18)$ & $14(4-22.5)^{\mathrm{a}}$ & $4(2-5)^{b}$ & $9.5(2-16.75)^{a, c}$ & $5(2-15.5)^{\mathrm{b}, \mathrm{c}}$ & $<0.001$ \\
\hline Psychological well-being before COVID-19, median (IQR) & $8(7-8)$ & $8(7-8)$ & $8(7-8)$ & $7(7-8)$ & $8(7-8.25)$ & 0.246 \\
\hline Underwent a quarantine period, $n(\%)$ & $51(10.1)$ & $27(14.6)$ & $8(7.2)$ & $5(6.1)$ & $11(8.8)$ & 0.079 \\
\hline Experienced a period of self-isolation, $n(\%)$ & $161(32.2)$ & $84(45.4)^{\mathrm{a}}$ & $24(21.6)^{b}$ & $23(28.0)^{b}$ & $31(24.8)^{\mathrm{b}}$ & $<0.001$ \\
\hline Experience of stressful events related to COVID-19, $n$ (\%) & $261(51.9)$ & $114(61.6)^{\mathrm{a}}$ & $40(36.0)^{b}$ & $35(42.7)^{b, c}$ & $72(57.6)^{\mathrm{a}, \mathrm{c}}$ & $<0.001$ \\
\hline Experience of stressful events not related to COVID-19, $n(\%)$ & $118(23.5)$ & $49(26.5)$ & $18(16.2)$ & $18(22.0)$ & $33(26.4)$ & 0.180 \\
\hline Perceived risk of being infected, median (IQR) & $6(5-8)$ & $7(5-8)$ & $6(4-8)$ & $6(5-7)$ & $6(4-8)$ & 0.055 \\
\hline Perceived risk of death in case of infection, median (IQR) & $3(2-4)$ & $3(2-5)$ & $3(2-4)$ & $3(2-5)$ & $3(2-5)$ & 0.483 \\
\hline
\end{tabular}

COVID-19, coronavirus disease 2019; IQR, interquartile range. Each subscript letter denotes a subset of University hospital categories whose parameters do not differ significantly from each other at the 0.05 level.

information criterion, did not show a statistically significant improvement of the model fit using any multilevel logistic regression model; therefore, we maintained the multivariable logistic regression model with fixed coefficients as it was more parsimonious.

\section{Association Between Psychological Distress and Perception of HCPs of Aspects Related to the Greatest Stress}

The perception of respondents regarding the aspects associated with the greatest stress is shown in Table 5. The fear of infecting the family and the continuous updating of recommendations and measures to be implemented were the most perceived distressing factors. These two aspects related to distress were reported by $56.8 \%$ of respondents. However, they were not associated with the GHQ-12 score. The constant and correct use of PPE was the third most frequent aspect related to the greatest stress. It was reported with higher frequency by HCPs in the group having GHQ-12<3. Conversely, a significantly higher proportion of HCPs in the group having GHQ-12 $\geq 3$ reported difficulties in reconciling private and family life with work, although this aspect was indicated by only $11.6 \%$ of HCPs.

\section{Association Between Psychological Distress and Perception of HCPs of Interpersonal and Organizational Factors}

Regarding interpersonal and organizational factors at work (Table 6), the group with psychological distress (GHQ-12 $\geq 3$ ) reported more irritability in the relationship with the patient and guilt about the poor chance of collaboration. The contrary emerged for feelings of group cohesion. For the group having GHQ-12 $\geq 3$, colleagues' support was more frequently reported as a factor that helped to face the emergency at work than in those without the evidence of clinically significant psychological distress (GHQ-12<3).

\section{DISCUSSION}

\section{Prevalence of Psychological Distress}

Half of the HCPs who completed the GHQ-12 reported a clinically significant level of psychological distress (Piccinelli et al., 1993). This result is consistent with previous Italian and international studies exploring the psychological impact of the COVID-19 pandemic on HCPs 


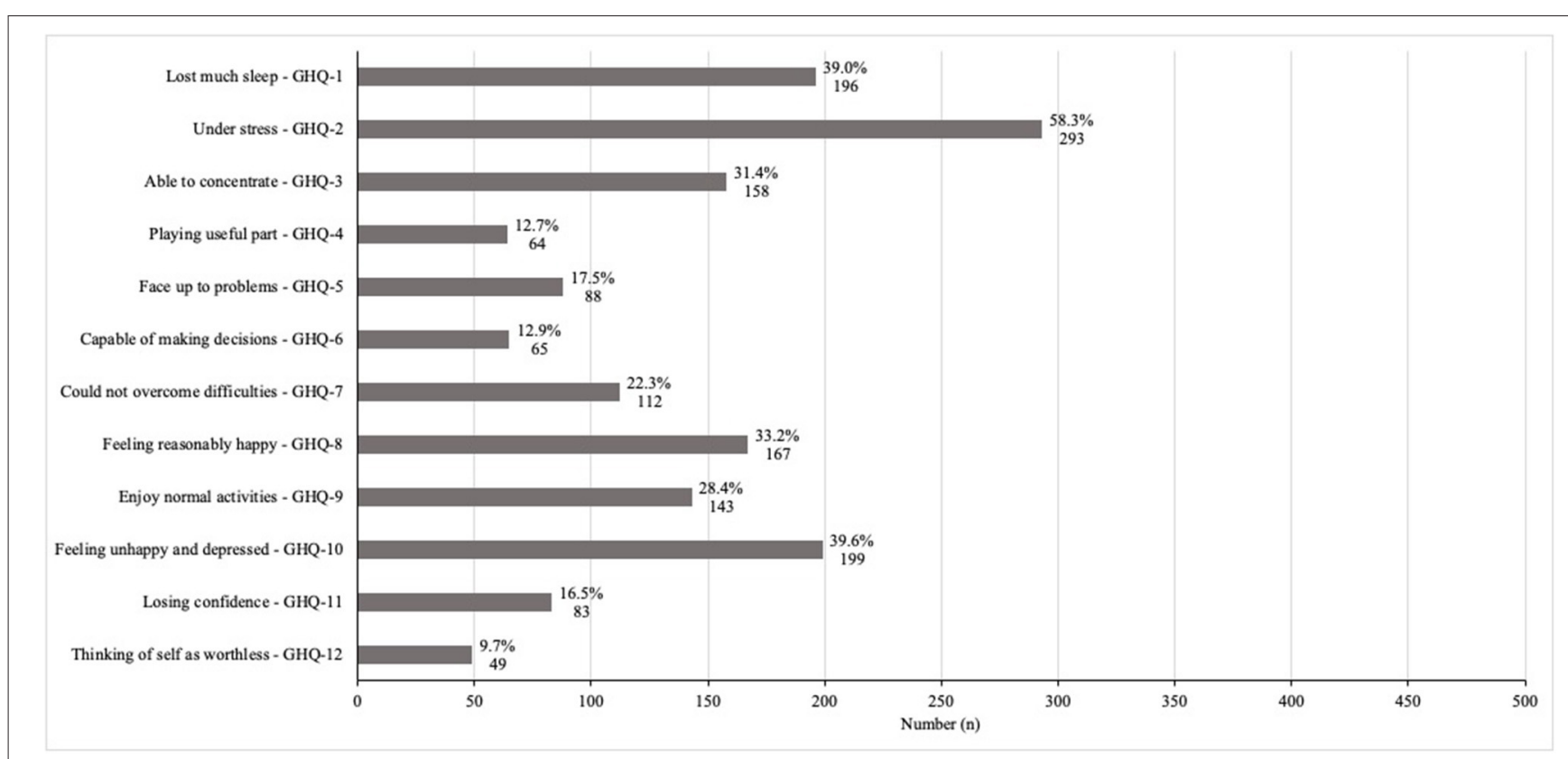

FIGURE 1 | General health and professional well-being assessed with the 12-item General Health Questionnaire (GHQ-12). The proportion of patients with a positive score for a worsening in each item composing the GHQ-12 $(n=481)$.

(Barello et al., 2020a,b; Galli et al., 2020; Giusti et al., 2020; Lai et al., 2020; Nie et al., 2020).

To the best of our knowledge, only one previous study in Europe specifically investigated this topic among HCPs working in obstetrics. Our results confirm the observed high level of psychological distress, although the percentage of HCPs with GAD-2 and PHQ-2 questionnaires scores suggestive of anxiety and depressive disorders was lower in the UK-based study than in our study (i.e., respectively, 25 and 16\%) (Shah et al., 2020). However, the comparison with some of the prior studies is limited by using different psychological screening instruments or scoring methods for the GHQ-12. When comparing our results with the studies using the same GHQ-12 scoring system, we observed that the percentage of HCPs with clinically significant psychological distress was similar to that reported in a study conducted in China during the pandemic outbreak (Yao et al., 2020). Moreover, in both studies, "being under stress" and "having lost much sleep" were the GHQ-12 items most negatively affected (Yao et al., 2020). Noteworthy, in our sample, around $40 \%$ of HCPs reported being "feeling unhappy and depressed" rather or much more than usual.

Based on our results and previous evidence, psychological screening appears necessary to recognize psychological suffering and prevent negative consequences on HCPs and patient care in obstetrics. Notably, psychological support for these HCPs was already recommended before the current pandemic, considering that gynecologists and midwives are known to be exposed to high levels of posttraumatic stress disorder (Wahlberg et al., 2017; Bourne et al., 2019; Slade et al., 2020).

\section{Factors Associated With Psychological Distress at the Individual, Interpersonal, and Organizational Levels}

In our analysis, the identified model (socioecological) (Winkel et al., 2019), explaining the psychological distress among HCPs in obstetrics, included factors at individual, interpersonal, and organizational levels. Based on the multilevel regression analysis, the relevance of these factors appears similar across the included hospitals. These results reinforce the need to intervene at different levels to reduce the risk of psychological distress in dealing with the COVID-19 pandemic (Winkel et al., 2019; Slade et al., 2020).

\section{Role of Individual Factors}

During the pandemic, being female was one of the main factors associated with psychological distress in our model, similar to previous studies in obstetrics (Shah et al., 2020) or other HCP categories (Babore et al., 2020; Barello et al., 2020a; Di Tella et al., 2020; Shaukat et al., 2020; Yao et al., 2020). This result is coherent with a higher level of mental and stress disorders observed during the COVID-19 pandemic among females of the general population. This higher vulnerability of females to experience stress and develop posttraumatic symptoms was explained by differences in stress-response systems and a higher involvement as family caregivers (Mazza et al., 2020; GarcíaFernández et al., 2021). However, the higher prevalence of anxiety and mood disorders in females is recognized in many epidemiological studies (Kessler et al., 2005). Different biological, psychological, social, and gender-role theoretical explanations have been proposed to explain these differences. 
TABLE 2 | Univariate logistic analysis of factors evaluated for an association with GHQ-12 $\geq 3(n=481)$.

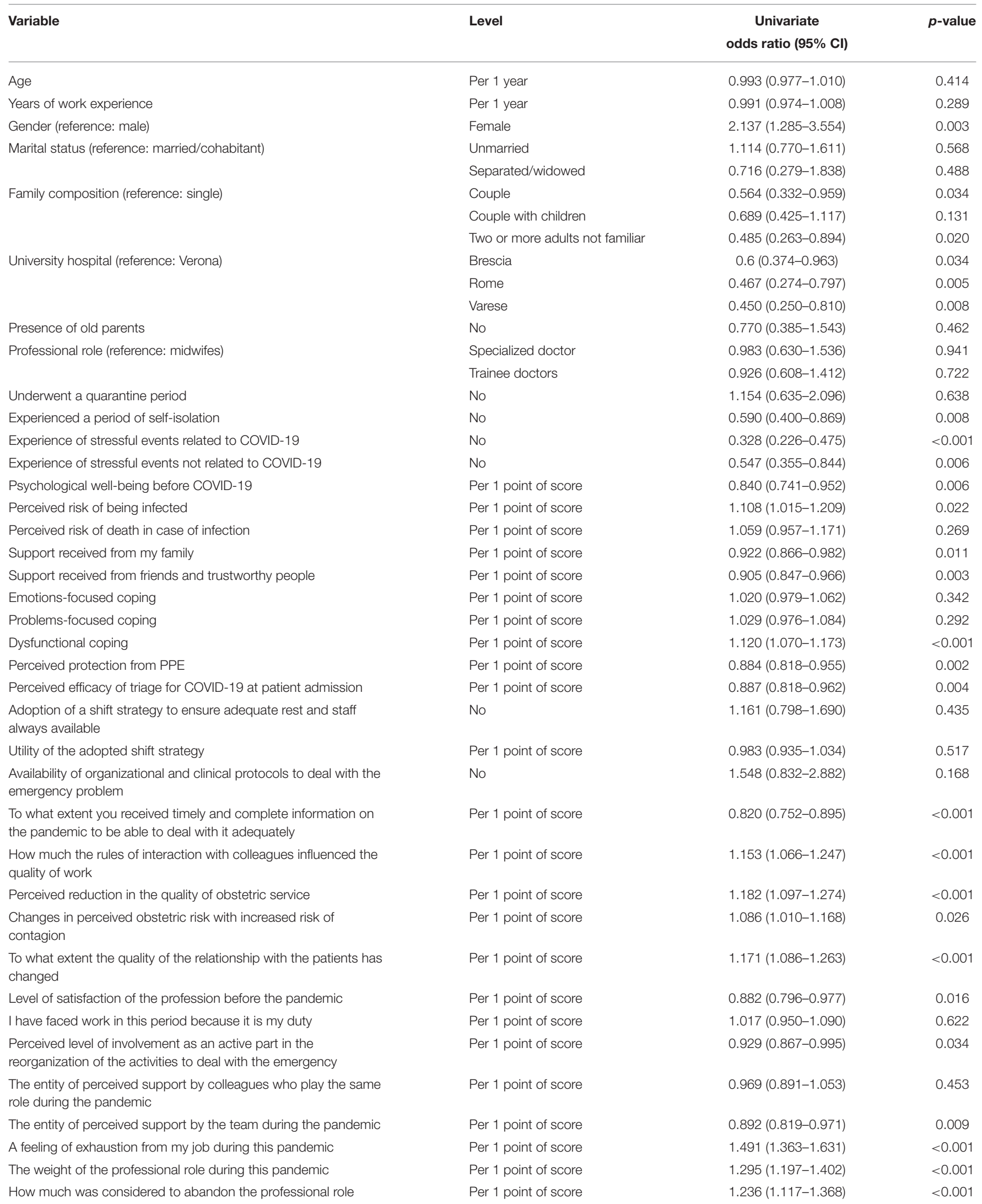


TABLE 3 | Details regarding the Brief-COPE items composing the dysfunctional coping factor.

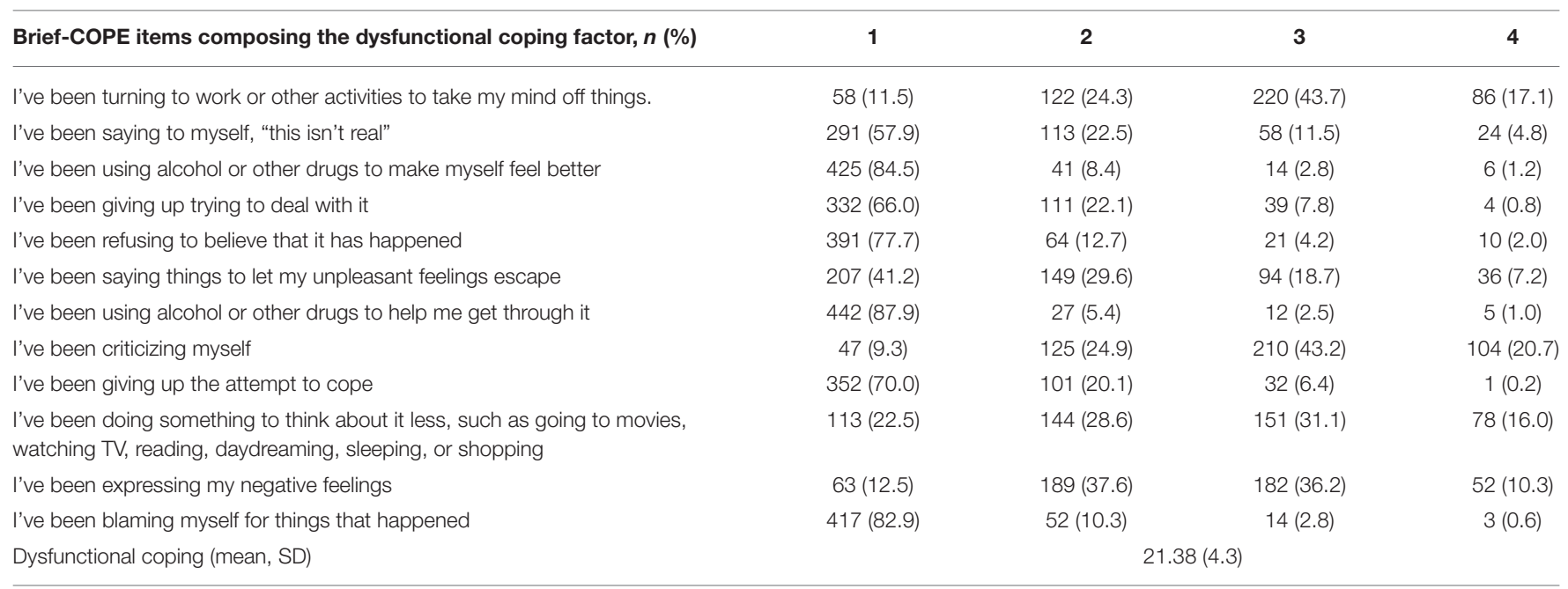

SD, standard deviation. All healthcare provides answered the Brief-COPE; each item provides a score from 1 to 4 , which is summed to obtain the dysfunctional coping score.

TABLE 4 | Multivariable logistic regression model of factors evaluated for an association with GHQ-12 $\geq 3(n=481)$.

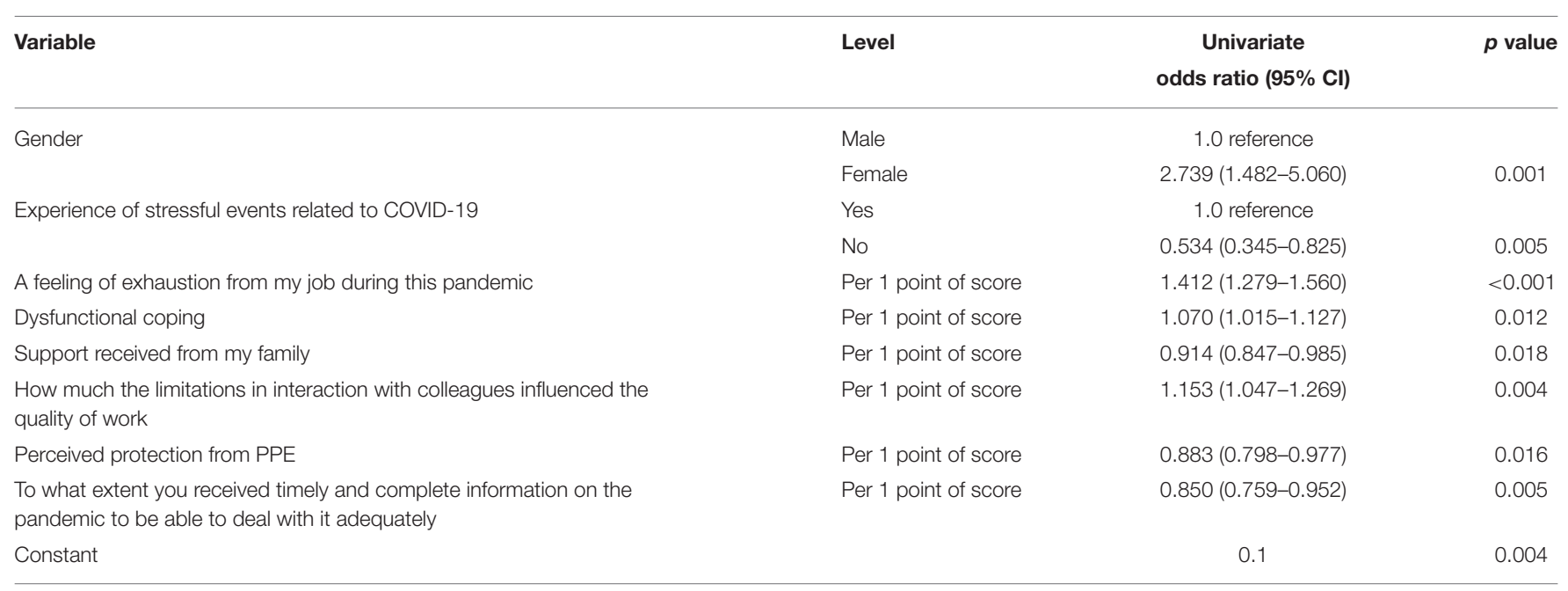

$R^{2}=0.279$ (Cox and Snell), 0.372 (Nagelkerke).

Among other individual factors, the professional role and fewer years of experience were not associated with psychological distress in our sample, which is discordant with the previous studies (Barello et al., 2020b; Kisely et al., 2020; Marton et al., 2020; Yao et al., 2020). Conversely, we found that a lower level of self-evaluated psychological well-being before the pandemic was related to psychological distress in univariate analysis. This association was in line with some previous studies in which having a prior history of psychological distress has been considered a vulnerability factor during virus outbreaks (Giusti et al., 2020; Kisely et al., 2020).

Stressful events related to COVID-19 remained a factor associated with psychological distress in the multivariable analysis. Even non-frontline HCPs, such as gynecologists and midwives, experienced highly stressful situations, including quarantine and self-isolation. Notably, consistent with a previous survey in obstetrics (Yalçin Bahat et al., 2020) and HCPs in Italy (Marton et al., 2020), "fear to infect my family" was among the major sources of perceived stress, despite the fact that this fear was not associated with psychological distress.

In terms of coping, higher use of dysfunctional coping strategies was associated with clinically significant psychological distress. This is consistent with previously reported association between avoidant coping strategies and burnout and lower compassion satisfaction in the medical setting (Doolittle, 2020). Moreover, avoidant coping was associated with the perceived stress during the pandemic in an Italian study (Babore et al., 2020). The dysfunctional coping strategies mostly frequently used by our study participants were self-blame but also self-distraction strategies, such as doing something to think about the pandemic less. Notably, in emergency and uncertain conditions such as the COVID-19 pandemic, self-distraction might be considered 
TABLE 5 | Association between psychological distress and perception of HCPs of aspects related to the greatest stress $(n=481)$.

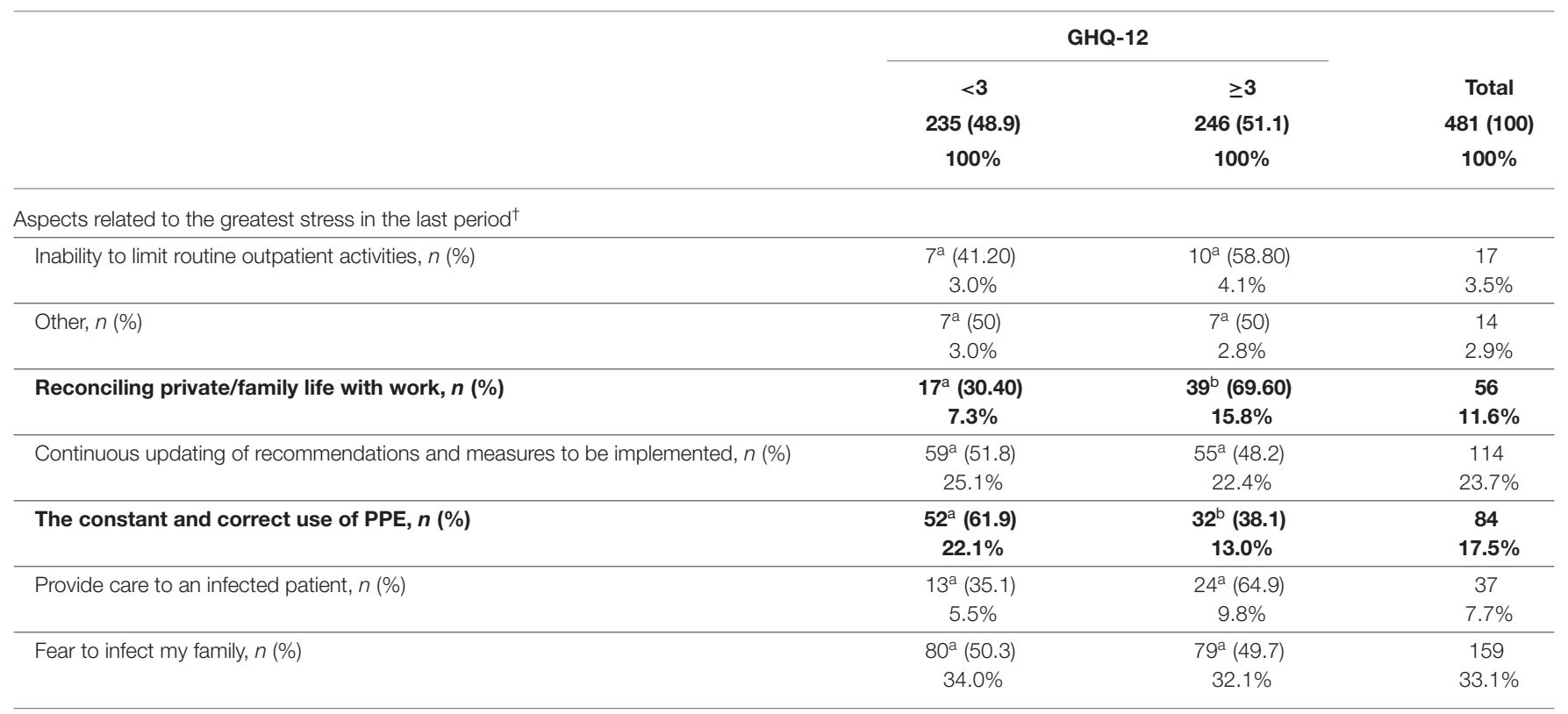

$\chi^{2}$ Test regarding the entire table $=0.009$. In bold significant associations. Each subscript letter denotes a subset of GHQ-12 categories whose column proportions do not differ significantly from each other at the 0.05 level.

†' Single answer allowed.

psychologically protective when used as a short-term strategy, although it might become problematic in the long term.

\section{Role of Interpersonal Factors}

Higher perceived support by the family was associated with a lower prevalence of psychological distress, in line with previous research among HCPs working in obstetrics (Vafaei et al., 2020; Yalçin Bahat et al., 2020) and other fields (Di Tella et al., 2020; Galli et al., 2020; Kisely et al., 2020; Nie et al., 2020). Our study supports these findings as our participants with clinically significant psychological distress reported twice as those without significant distress "reconciling private and family life with work" as one of the aspects related to the greatest stress.

Moreover, the significance of relationships with colleagues was confirmed as a relevant resilience feature in the obstetrics context (Winkel et al., 2019). Reduced interactions with colleagues were associated with psychological distress. In addition, HCPs presenting with psychological distress showed a higher frequency of "guilt about my poor chance of collaboration" and lower "perception of group cohesion" than the counterparts.

\section{Role of Organizational Factors}

In line with previous research (Green et al., 2020; Kisely et al., 2020; Nie et al., 2020; Semaan et al., 2020), higher scores in "perceived protection by PPE" and "receiving timely and complete information on the pandemic to deal with it" were associated with lower psychological distress. The relevance of information and PPE on psychological distress in our sample may be intensified because Italy was one of the first countries to face the pandemic. This phase was characterized by continuously changing guidelines and protocols and problematic resource allocation, including PPE, resulting in the exposure of HCPs to safety risk and psychological pressure (Oliva et al., 2020).

Notably, in our sample, HCPs perceived "continuous updating of recommendations and measures to be implemented" as the second aspect related to the greatest stress. This was also a major factor associated with the mental health status in the UK survey on HCPs in obstetrics (Shah et al., 2020).

\section{Strengths and Limitations}

The main strengths of the present study are the high response rate and the inclusion of both gynecologists-whether already specialized or trainees-and midwives. These characteristics allowed building a comprehensive and representative picture of the psychological impact of the COVID-19 pandemic in the obstetrics field. Second, an extensive list of potentially associated factors has been considered in our survey, in line with the conceptualization of psychological distress as a complex interaction between individual, interpersonal, and organizational factors (Winkel et al., 2019). Third, the survey items were created by a panel of experts in the field (i.e., HCPs working in the obstetrics and clinical psychologists supporting hospital HCPs during the pandemic), favoring the feasibility and multidisciplinary nature of the survey. Lastly, the use of validated instruments, such as the GHQ-12, a widely used and validated tool for screening psychiatric morbidity, increases generalizability of our findings and reproducibility of our study by other centers (Goldberg et al., 1997; Werneke et al., 2000).

One of the main limitations to the present study is the cross-sectional design. This study design is not appropriate for determination of causal effect and also limits the ability to explore temporal association and their variations over time. 
TABLE 6 | Association between psychological distress and perception of HCPs of interpersonal and organizational factors $(n=481)$.

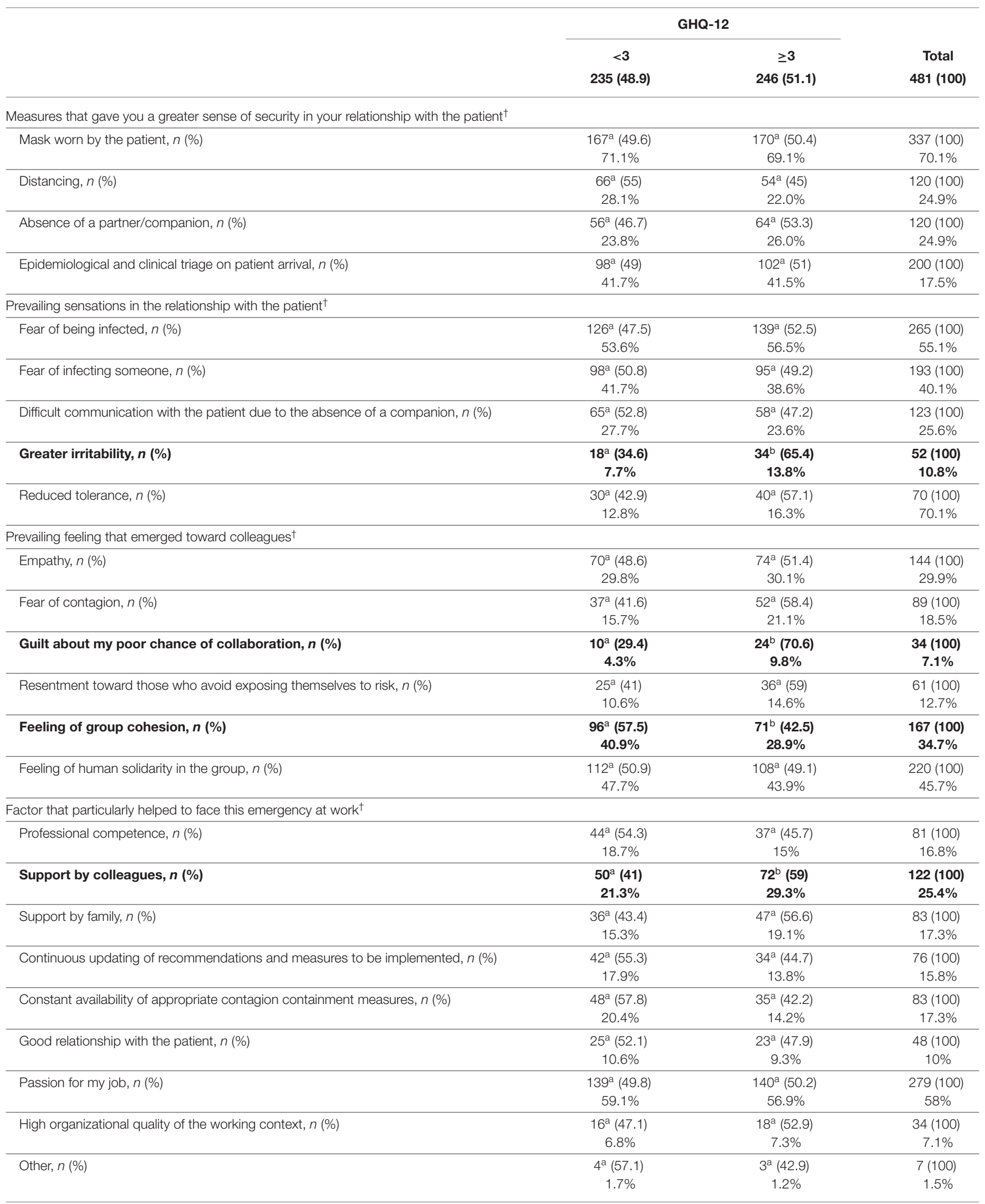

In bold significant associations. Each subscript letter denotes a subset of GHQ-12 categories whose column proportions do not differ significantly from each other at the 0.05 level. ${ }^{\dagger}$ Multiple answers were allowed. 
Psychological distress and other HCPs' perceptions and emotions were self-reported simultaneously at a single time point. Therefore, we cannot determine which is a cause and which a consequence of psychological distress. On that basis, further longitudinal studies should be conducted to verify observed results and clarify temporal associations.

Additional limitations related to the cross-sectional design are also present. A non-respondent bias should be considered, although a response rate of $84.4 \%$ reduces its impact. A possible referral bias suggests caution in extending our observations to HCPs in obstetrics who do not work in hospitals. In this regard, the generalizability of results may be affected by differences in infection risk and healthcare management of the outbreak across Italy (Armocida et al., 2020; Simione and Gnagnarella, 2020) or worldwide. In terms of geographical context, indeed, HCPs working in regions most affected by the pandemic reported a higher negative psychological impact (Trumello et al., 2020). However, our results suggest that the proposed model does not change across investigated hospitals, given that the multilevel regression analysis did not provide a better model fit in describing the outcome. A recall bias can be present for some items, such as the psychological well-being before the pandemic.

As an additional limitation, the evaluation of "enjoyment of day-to-day activities" of the GHQ-12 may be affected by the Italian lockdown as previously outlined in a UK article (Niedzwiedz et al., 2020). Moreover, our study focused on assessing the psychological distress in the aftermath of the pandemic. Therefore, future studies are required to determine the long-lasting psychological effects and the potential impact on burnout.

\section{CONCLUSIONS}

The psychological well-being of HCPs working in obstetrics at four Italian hospitals was poor during the COVID-19 outbreak, given that just over half of the respondents who reported clinically significant psychological distress. This observation stresses the importance of introducing a psychological screening and enhancing individuals and interpersonal and organizational resources to face stressful events, such as a pandemic. At the individual level, psychological interventions should promote acceptance of negative emotions and reduction of avoidance strategies and self-blame and should improve debriefing of stressful experiences. The crucial role of interpersonal factors suggested that group interventions, such as daily experience sharing and peer support, might be effective strategies aimed at normalizing and reducing psychological distress and the perceived difficulties in reconciling private and family life with work. Implementing group initiatives might also enhance the peer recognition of more vulnerable HCPs and reduce stigma. However, at the same time, actions at the organizational level are mandatory to ensure timely and complete access to information and proper material resources, such as PPE. Moreover, at this higher level, a culture of collaboration and support is essential to enhance actions at the individual and interpersonal level, as already suggested for the obstetrics context (Slade et al., 2020). Enhancing these integrated strategies may reduce the psychological impact of COVID-19 and other pandemics and mitigate the potential adverse effects of severe obstetric events, which remain a major source of work-related stress disorders.

\section{DATA AVAILABILITY STATEMENT}

The raw data supporting the conclusions of this article are available from the corresponding author on reasonable request.

\section{ETHICS STATEMENT}

The study involving human participants was reviewed and approved by the Human research ethics committee of the University of Verona (CARU, Comitato di Approvazione della Ricerca sull'Uomo) - 2020-UNVRCLE-0143469. The patients/participants provided their informed consent to participate in this study.

\section{AUTHOR CONTRIBUTIONS}

MF, RR, LD, VD, MR, CP, FG, ES, and GS conceptualized and designed the study. LD, RR, MF, VD, MR, and CP developed the questionnaire. SU, AC, FG, MG, ES, GS, and FC organized and performed the survey. SG, SU, MG, and FC managed the dataset and performed statistical analyses. LD, VD, and SG wrote the manuscript. All the authors conform to the International Committee of Medical Journal Editors (ICMJE) criteria for authorship, contributed to the intellectual content of the study, approved the final version of the article, contributed to the interpretation of the results, and the writing and editing of the manuscript.

\section{ACKNOWLEDGMENTS}

The authors thank Dr. Marina Buciuc for the contribution to the language revision of the manuscript.

\section{REFERENCES}

Alfieri, N., Manodoro, S., and Marconi, A. M. (2020). COVID-19 does not stop obstetrics: what we need to change to go on safely birthing. The experience of a University obstetrics and gynecology department in Milan. J. Perinat. Med. 48, 997-1000. doi: 10.1515/jpm2020-0218
Armocida, B., Formenti, B., Ussai, S., Palestra, F., and Missoni, E. (2020). The Italian health system and the COVID-19 challenge. Lancet Public Health 5:e253. doi: 10.1016/S2468-2667(20)30074-8

Babore, A., Lombardi, L., Viceconti, M. L., Pignataro, S., Marino, V., Crudele, M., et al. (2020). Psychological effects of the COVID-2019 pandemic: perceived stress and coping strategies among healthcare professionals. Psychiatry Res. 293:113366. doi: 10.1016/j.psychres.2020.113366 
Barello, S., Palamenghi, L., and Graffigna, G. (2020a). Burnout and somatic symptoms among frontline healthcare professionals at the peak of the Italian COVID-19 pandemic. Psychiatry Res. 290:113129. doi: $10.1016 /$ j.psychres.2020.113129

Barello, S., Palamenghi, L., and Graffigna, G. (2020b). Stressors and resources for healthcare professionals during the Covid-19 pandemic: lesson learned from Italy. Front. Psychol. 11:2179. doi: 10.3389/fpsyg.2020.02179

Becker, J. L., Milad, M. P., and Klock, S. C. (2006). Burnout, depression, and career satisfaction: cross-sectional study of obstetrics and gynecology residents. Am. J. Obstet. Gynecol. 195, 1444-1449. doi: 10.1016/j.ajog.2006.06.075

Boelig, R. C., Saccone, G., Bellussi, F., and Berghella, V. (2020). MFM guidance for COVID-19. Am. J. Obstet. Gynecol. MFM 2:100106. doi: 10.1016/j.ajogmf.2020.100106

Bourne, T., Shah, H., Falconieri, N., Timmerman, D., Lees, C., Wright, A., et al. (2019). Burnout, well-being and defensive medical practice among obstetricians and gynaecologists in the UK: cross-sectional survey study. BMJ Open 9:e030968. doi: 10.1136/bmjopen-2019-030968

Carver, C. S. (1997). You want to measure coping but your protocol's too long: consider the brief COPE. Int. J. Behav. Med. 4, 92-100. doi: 10.1207/s15327558ijbm0401_6

Carver, C. S., Scheier, M. F., and Weintraub, J. K. (1989). Assessing coping strategies: a theoretically based approach. J. Pers. Soc. Psychol. 56, 267-283. doi: 10.1037/0022-3514.56.2.267

Chowdhury, M. Z. I., and Turin, T. C. (2020). Variable selection strategies and its importance in clinical prediction modelling. Fam. Med. Commun. Health 8:e00262. doi: 10.1136/fmch-2019-000262

Cohen, J., Cohen, P., West, S. G., and Aiken, L. S. (2013). Applied Multiple Regression/Correlation Analysis for the Behavioral Sciences. New York, NY: Routledge, 1-900. doi: 10.4324/9780203774441

Coolidge, F. L., Segal, D. L., Hook, J. N., and Stewart, S. (2000). Personality disorders and coping among anxious older adults. J. Anxiety Disord. 14, 157-172. doi: 10.1016/S0887-6185(99)00046-8

Del Piccolo, L., Raffaelli, R., Garzon, S., Bosco, M., Casarin, J., Ciccarone, F., et al. (2020). IPSICO survey on the psychological impact of COVID-19 on healthcare providers in obstetrics: a study design. Ital. J. Gynaecol. Obstet. 32, 276-286. doi: $10.36129 /$ jog. 32.04 .07

Dell'Utri, C., Manzoni, E., Cipriani, S., Spizzico, C., Dell'Acqua, A., Barbara, G., et al. (2020). Effects of SARS Cov-2 epidemic on the obstetrical and gynecological emergency service accesses. What happened and what shall we expect now? Eur. J. Obstet. Gynecol. Reprod. Biol. 254, 64-68. doi: 10.1016/j.ejogrb.2020.09.006

Di Tella, M., Romeo, A., Benfante, A., and Castelli, L. (2020). Mental health of healthcare workers during the COVID-19 pandemic in Italy. J. Eval. Clin. Pract. 26, 1583-1587. doi: 10.1111/jep.13444

Doolittle, B. R. (2020). Association of burnout with emotional coping strategies, friendship, and institutional support among internal medicine physicians. J. Clin. Psychol. Med. Settings 15, 1-7. doi: 10.1007/s10880-02009724-6

Franchi, M., Bosco, M., Garzon, S., Lagan,à A. S., Cromi, A., Barbieri, B., et al. (2020). Management of obstetrics and gynaecological patients with COVID-19. Ital. J. Gynaecol. Obstet. 32, 6-19. doi: 10.36129/jog.32.01.01

Galli, F., Pozzi, G., Ruggiero, F., Mameli, F., Cavicchioli, M., Barbieri, S., et al. (2020). A systematic review and provisional metanalysis on psychopathologic burden on health care workers of coronavirus outbreaks. Front. Psychiatry 11:568664. doi: 10.3389/fpsyt.2020.568664

García-Fernández, L., Romero-Ferreiro, V., Padilla, S., David López-Roldán, P., Monzó-García, M., and Rodriguez-Jimenez, R. (2021). Gender differences in emotional response to the COVID-19 outbreak in Spain. Brain Behav. 11:e01934. doi: 10.1002/brb3.1934

Giusti, E. M., Pedroli, E., D’Aniello, G. E., Stramba Badiale, C., Pietrabissa, G., Manna, C., et al. (2020). The psychological impact of the COVID19 outbreak on health professionals: a cross-sectional study. Front. Psychol. 11:1684. doi: 10.3389/fpsyg.2020.01684

Goldberg, D. P., Gater, R., Sartorius, N., Ustun, T. B., Piccinelli, M., Gureje, O., et al. (1997). The validity of two versions of the GHQ in the WHO study of mental illness in general health care. Psychol. Med. 27, 191-197. doi: $10.1017 /$ S0033291796004242
Govardhan, L. M., Pinelli, V., and Schnatz, P. F. (2012). Burnout, depression and job satisfaction in obstetrics and gynecology residents. Conn. Med. 76, 389-395.

Green, L., Fateen, D., Gupta, D., McHale, T., Nelson, T., and Mishori, R. (2020). Providing women's health care during COVID-19: Personal and professional challenges faced by health workers. Int. J. Gynaecol. Obstet. Off. Organ. Int. Fed. Gynaecol. Obstet. 151, 3-6. doi: 10.1002/ijgo.13313

Greenberg, N., Docherty, M., Gnanapragasam, S., and Wessely, S. (2020). Managing mental health challenges faced by healthcare workers during covid19 pandemic. BMJ 368:m1211. doi: 10.1136/bmj.m1211

Hosmer, D. W. Jr., Lemeshow, S., and Sturdivant, R. X. (2013). Applied Logistic Regression. John Wiley \& Sons, 1-528. doi: 10.1002/9781118445112.stat06902

Kessler, R. C., Demler, O., Frank, R. G., Olfson, M., Pincus, H. A., Walters, E. E., et al. (2005). Prevalence and treatment of mental disorders, 1990 to 2003. N. Engl. J. Med. 352, 2515-2523. doi: 10.1056/NEJMsa043266

Kisely, S., Warren, N., McMahon, L., Dalais, C., Henry, I., and Siskind, D. (2020). Occurrence, prevention, and management of the psychological effects of emerging virus outbreaks on healthcare workers: rapid review and metaanalysis. BMJ 369:m1642. doi: 10.1136/bmj.m1642

Lai, J., Ma, S., Wang, Y., Cai, Z., Hu, J., Wei, N., et al. (2020). Factors associated with mental health outcomes among health care workers exposed to coronavirus disease 2019. JAMA Netw. Open 3:e203976. doi: 10.1001/jamanetworkopen.2020.3976

Marton, G., Vergani, L., Mazzocco, K., Garassino, M. C., and Pravettoni, G. (2020). 2020s heroes are not fearless: the impact of the COVID-19 pandemic on wellbeing and emotions of Italian health care workers during italy phase 1 . Front. Psychol. 11:588762. doi: 10.3389/fpsyg.2020.588762

Mazza, C., Ricci, E., Biondi, S., Colasanti, M., Ferracuti, S., Napoli, C., et al. (2020). A nationwide survey of psychological distress among italian people during the COVID-19 pandemic: immediate psychological responses and associated factors. Int. J. Environ. Res. Public Health 17:3165. doi: 10.3390/ijerph17093165

Nie, A., Su, X., Zhang, S., Guan, W., and Li, J. (2020). Psychological impact of COVID-19 outbreak on frontline nurses: a cross-sectional survey study. J. Clin. Nurs. 29, 4217-4226. doi: 10.1111/jocn.15454

Niedzwiedz, C. L., Green, M. J., Benzeval, M., Campbell, D., Craig, P., Demou, E., et al. (2020). Mental health and health behaviours before and during the initial phase of the COVID-19 lockdown: longitudinal analyses of the UK Household Longitudinal Study. J. Epidemiology. Commun. Health. 75, 224-231. doi: $10.1101 / 2020.06 .21 .20136820$

Oliva, A., Caputo, M., Grassi, S., Vetrugno, G., Marazza, M., Ponzanelli, G., et al. (2020). Liability of health care professionals and institutions during COVID-19 pandemic in Italy: symposium proceedings and position statement. J. Patient Saf. 16, e299-e302. doi: 10.1097/PTS.0000000000000793

Piccinelli, M., Bisoffi, G., Bon, M. G., Cunico, L., and Tansella, M. (1993). Validity and test-retest reliability of the Italian version of the 12-item general health questionnaire in general practice: a comparison between three scoring methods. Compr. Psychiatry 34, 198-205. doi: 10.1016/0010-440X(93)90048-9

Politi, P. L., Piccinelli, M., and Wilkinson, G. (1994). Reliability, validity and factor structure of the 12-item general health questionnaire among young males in Italy. Acta Psychiatr. Scand. 90, 432-437. doi: 10.1111/j.1600-0447.1994.tb01620.x

Preti, E., Di Mattei, V., Perego, G., Ferrari, F., Mazzetti, M., Taranto, P., et al. (2020). The psychological impact of epidemic and pandemic outbreaks on healthcare workers: rapid review of the evidence. Curr. Psychiatry Rep. 22:43. doi: 10.1007/s11920-020-01166-z

Qiao, J. (2020). What are the risks of COVID-19 infection in pregnant women? Lancet Lond. Engl. 395, 760-762. doi: 10.1016/S0140-6736(20)30365-2

Ratner, B. (2010). Variable selection methods in regression: ignorable problem, outing notable solution. J. Target Meas. Anal. Mark. 18, 65-75. doi: $10.1057 /$ jt.2009.26

Raudenbush, S. W., and Bryk, A. S. (2002). Hierarchical Linear Models: Applications and Data Analysis Methods. Chicago, IL: Sage, 1-520.

Semaan, A., Audet, C., Huysmans, E., Afolabi, B., Assarag, B., BankeThomas, A., et al. (2020). Voices from the frontline: findings from a thematic analysis of a rapid online global survey of maternal and newborn health professionals facing the COVID-19 pandemic. BMJ Glob. Health. 5:e002967. doi: 10.1101/2020.05.08.200 93393 
Shah, N., Raheem, A., Sideris, M., Velauthar, L., and Saeed, F. (2020). Mental health amongst obstetrics and gynaecology doctors during the COVID-19 pandemic: results of a UK-wide study. Eur. J. Obstet. Gynecol. Reprod. Biol. 253, 90-94. doi: 10.1016/j.ejogrb.2020.07.060

Shaukat, N., Ali, D. M., and Razzak, J. (2020). Physical and mental health impacts of COVID-19 on healthcare workers: a scoping review. Int. J. Emerg. Med. 13:40. doi: $10.1186 / \mathrm{s} 12245-020-00299-5$

Shreffler, J., Petrey, J., and Huecker, M. (2020). The impact of COVID-19 on healthcare worker wellness: a scoping review. West. J. Emerg. Med. 21, 1059-1066. doi: 10.5811/westjem.2020.7.48684

Simione, L., and Gnagnarella, C. (2020). Differences between health workers and general population in risk perception, behaviors, and psychological distress related to COVID-19 spread in Italy. Front. Psychol. 11:2166. doi: $10.3389 /$ fpsyg. 2020.02166

Slade, P., Balling, K., Sheen, K., Goodfellow, L., Rymer, J., Spiby, H., et al. (2020). Work-related post-traumatic stress symptoms in obstetricians and gynaecologists: findings from INDIGO, a mixed-methods study with a crosssectional survey and in-depth interviews. BJOG Int. J. Obstet. Gynaecol. 127, 600-608. doi: 10.1111/1471-0528.16076

Tabachnick, B. G., Fidell, L. S., and Ullman, J. B. (2019). Using Multivariate Statistics. Pearson, 1

Trumello, C., Bramanti, S. M., Ballarotto, G., Candelori, C., Cerniglia, L., Cimino, S., et al. (2020). Psychological adjustment of healthcare workers in Italy during the COVID-19 pandemic: differences in stress, anxiety, depression, burnout, secondary trauma, and compassion satisfaction between frontline and non-frontline professionals. Int. J. Environ. Res. Public Health. 17:8358. doi: 10.3390/ijerph17228358

Vafaei, H., Roozmeh, S., Hessami, K., Kasraeian, M., Asadi, N., Faraji, A., et al. (2020). Obstetrics healthcare providers' mental health and quality of life during COVID-19 pandemic: multicenter study from eight cities in Iran. Psychol. Res. Behav. Manag. 13, 563-571. doi: 10.2147/PRBM.S256780

van Oyen, H. (2009). Multilevel analysis of survey data. Int. J. Public Health 54, 129-130. doi: 10.1007/s00038-009-7075-z

Wahlberg, A., Andreen Sachs, M., Johannesson, K., Hallberg, G., Jonsson, M., Skoog Svanberg, A., et al. (2017). Post-traumatic stress symptoms in Swedish obstetricians and midwives after severe obstetric events: a cross-sectional retrospective survey. BJOG Int. J. Obstet. Gynaecol. 124, 1264-1271. doi: 10.1111/1471-0528.14259

Werneke, U., Goldberg, D. P., Yalcin, I., and Ustün, B. T. (2000). The stability of the factor structure of the general health questionnaire. Psychol. Med. 30, 823-829. doi: $10.1017 /$ S0033291799002287

WHO (2020). Director-General's Opening Remarks at the Media Briefing on COVID. Available online at: https://www.who.int/director-general/speeches/ detail/who-director-general-s-opening-remarks-at-the-media-briefing-oncovid-19-\$-\$11-march-2020 (accessed January 2, 2021).

Winkel, A. F., Robinson, A., Jones, A.-A., and Squires, A. P. (2019). Physician resilience: a grounded theory study of obstetrics and gynaecology residents. Med. Educ. 53, 184-194. doi: 10.1111/medu.13737

Yalçin Bahat, P., Aldikaçtioglu Talma,ç M., Bestel, A., Topbas Selcuki, N. F., Karadeniz, O., and Polat, I. (2020). Evaluating the effects of the COVID19 pandemic on the physical and mental well-being of obstetricians and gynecologists in Turkey. Int. J. Gynaecol. Obstet. Off. Organ. Int. Fed. Gynaecol. Obstet. 151, 67-73. doi: 10.1002/ijgo.13287

Yao, Y., Tian, Y., Zhou, J., Diao, X., Cao, B., Pan, S., et al. (2020). Psychological status and influencing factors of hospital medical staff during the COVID-19 outbreak. Front. Psychol. 11:1841. doi: 10.3389/fpsyg.2020. 01841

Conflict of Interest: The authors declare that the research was conducted in the absence of any commercial or financial relationships that could be construed as a potential conflict of interest.

Copyright @ 2021 Del Piccolo, Donisi, Raffaelli, Garzon, Perlini, Rimondini, Uccella, Cromi, Ghezzi, Ginami, Sartori, Ciccarone, Scambia and Franchi. This is an openaccess article distributed under the terms of the Creative Commons Attribution License (CC BY). The use, distribution or reproduction in other forums is permitted, provided the original author(s) and the copyright owner(s) are credited and that the original publication in this journal is cited, in accordance with accepted academic practice. No use, distribution or reproduction is permitted which does not comply with these terms. 